\title{
Spontaneous modulation instability in noninstantaneous self-defocusing medium with a coherent feedback
}

\author{
Wen-Han Chu, Ming-Feng Shih* \\ Department of Physics, National Taiwan University, 1 Roosevelt Road, Section 4, Taipei 106, Taiwan
}

\section{A R T I C L E I N F O}

\section{Article history:}

Received 8 December 2008

Received in revised form 26 February 2009

Accepted 26 February 2009

\section{Keywords:}

Modulation instability

Noninstantaneous

Self-defocusing

Coherent feedback

\begin{abstract}
A B S T R A C T
We find theoretically that the optical modulation instability can spontaneously happen in the noninstantaneous defocusing medium when there is a coherent feedback. It requires both above-threshold gain and phase matching for the instability to happen, very similar to the oscillation condition of a laser. The theoretical result is in good agreement with our experimental observation.
\end{abstract}

(C) 2009 Elsevier B.V. All rights reserved.

\section{Introduction}

Modulation instability (MI), which happens in many nonlinear wave systems [1-3], is a phenomenon that a small amplitude modulation of a carrier wave grows exponentially due to the nonlinear response of the medium. In tradition, it is believed that spatial optical MI can only exist in the self-focusing medium since the so-called Lighthill criterion, requiring the nonlinearity working opposite to the diffraction, must be satisfied [3,4]. However, it has been known that the behavior of the system may be modified, or even is completely different, if the response of the system is delayed as compared to the instantaneously responding system [58]. In the former study [9-11], it is demonstrated that MI may still occur in the self-defocusing medium in the noninstantaneously delayed defocusing medium and the growth rate is a function of the spatial and temporal frequencies of the input perturbation (Fig. 1). In Fig. 1, the instability gain coefficient has no peaks, different from the usual gain coefficient for self-focusing medium, in which the spontaneous MI patterns always happen at the frequency of the highest gain [1-4]. As a result, if the light contains only white noise, every component at the gain plateau cannot single itself out and the pattern cannot form spontaneously. Recently, it has been demonstrated that in a cavity, the characteristic of MI is greatly modified [12-15]. Other than that, pattern formations with two light beams counter propagating in a nonlinear medium [1619] or with the liquid crystal light valve put into a ring cavity [20-

\footnotetext{
* Corresponding author. Tel./fax: +886 233665181 .

E-mail address: mfshih@phys.ntu.edu.tw (M.-F. Shih).
}

22] have also shown intriguing behaviors induced by the feedback. In this paper, we will demonstrate theoretically that the spatial MI can spontaneously occur in the noninstantaneous defocusing medium when an appropriate ring coherent feedback is present, and demonstrate its dependence on the feedback, similar to the oscillation condition of a laser cavity, requiring above-threshold gain and phase matching. We also demonstrate the results experimentally and find it in good agreement with the theory.

\section{Theoretical analysis}

The analysis is based on the setup shown in Fig. 2. A beam splitter (BS), a polarization beam splitter (PBS1), and a pair of mirrors form the cavity. Inside the cavity, a noninstantaneous self-defocusing medium of length $L$ is placed. We first consider the field propagation in the defocusing medium. We follow similar steps in the previous research [11]. We start from $\nabla^{2} E-\mu\left(\partial^{2} D / \partial t^{2}\right)=0$, with $D=\epsilon_{r}\left(|E|^{2}\right) E$ being the displacement and the dielectric coefficient $\epsilon_{r}=\epsilon_{0}\left(n_{0}+\delta n\right)^{2}$. Assuming that the nonlinearity is of the relaxation type with time constant $\tau$, we have $\delta n\left[|E(t)|^{2}\right]=\int_{-\infty}^{t}(1 / \tau)$ $F\left[\left|E\left(t_{1}\right)\right|^{2}\right] \exp \left[-\left(t-t_{1}\right) / \tau\right] d t_{1}$, with $F$ being the nonlinearity form. Notice that when substituting $E=A(\vec{r}, t) \exp (i w t-i k z)$ into the differential equation above, we neglect all time derivatives of the envelope $A$ much smaller than the second time derivative of $\exp (i w t-i k z)$, if $1 / \tau \ll \omega$. Using the paraxial approximation, we obtain

$\nabla_{\perp}^{2} A-2 i k \frac{\partial A}{\partial z}+2 k^{2} \frac{\delta n\left(|A|^{2}\right)}{n_{0}} A=0$. 


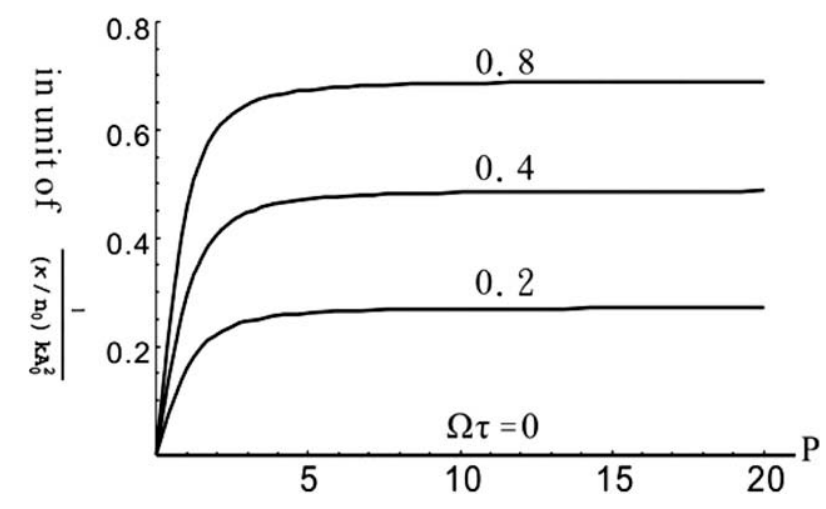

Fig. 1. Gain Spectrum of modulation instability in a self-defocusing medium. $P$ is the normalized spatial frequency, $\Omega$ the temporal frequency and $\tau$ the response time of the material.

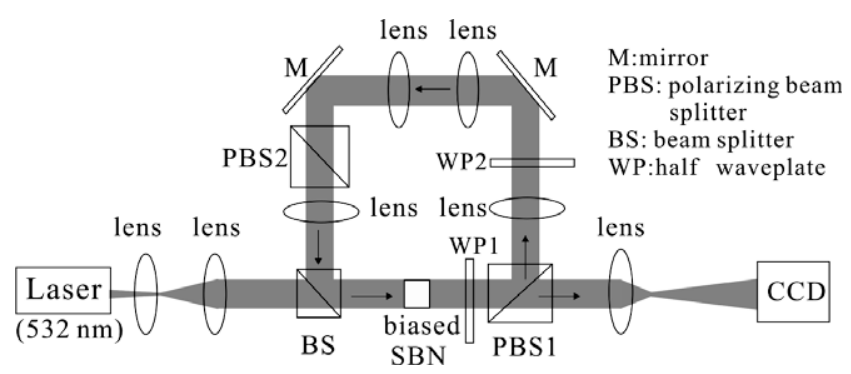

Fig. 2. Experimental setup.

Let $A=\left(A_{0}+a\right) \exp (-i \gamma) z$ as the unperturbed plane wave plus a small perturbation $a$, where $\gamma=k F\left(A_{0}^{2}\right) / n_{0}$ and $a=a_{1}+a_{2}$ with $a_{1,2}=\operatorname{Re}\left[a_{1,2}^{0} \exp \left(i \Omega \cdot t+i \vec{k}_{\perp} \cdot \vec{r}-i h_{1} z+h_{2} z\right)\right] . \Omega$ and $k_{\perp}$ are the temporal frequency and spatial frequency, respectively. By separating the real and imaginary parts of Eq. (1), we get $h_{1}=-\left[\frac{\kappa k}{n_{0}} A_{0}^{2}\right]^{2} \frac{Q}{1+Q} \frac{P^{2}}{h_{2}}$ with $h_{2}=\frac{\kappa k}{\sqrt{2} n_{0}} A_{0}^{2} \sqrt{-P^{4}-\frac{2 P^{2}}{1+Q^{2}}+P^{2} \sqrt{P^{4}+\frac{4+4 P^{2}}{1+Q^{2}}}}$. Here, $P=k_{\perp} /\left[\left(2 \kappa / n_{0}\right)\right]^{1 / 2} \cdot k A_{0}$ is the normalized spatial frequency, $Q=\Omega \tau$ the normalized temporal frequency, and $\kappa=\left.\frac{d F(I)}{d I}\right|_{I=A_{0}^{2}}$ the nonlinearity strength. The gain $h_{2}$ for several $Q(\Omega \tau)$ combinations is shown in Fig. 1. As the former study [11] has shown, the perturbation of light propagating through a distance L in a noninstantaneous self-defocusing medium will acquire a amplitude growth and a phase shift by $\exp \left(h_{2} L\right) \exp \left(i h_{1} L\right)$. We then consider for the feedback. We assume a fraction of light, $\epsilon$ in amplitude, exiting the medium is fed back to the input face of the medium. With careful optical setup, the image at the output face of the medium is mapped at the input face of the medium with exactly the same transverse coordinates. Light in the cavity can be treated as a single optical mean field when the coherence length of the light is longer than the round-trip length of the cavity [15]. For every Fourier component, $a(0)$, of any combination of spatial frequency and temporal frequency of the noise in the field entering the cavity, the same Fourier component $a(L)$ existing the nonlinear medium is

$a(L)=[a(0)+\epsilon a(L)] \exp \left(h_{2} L\right) \exp \left(i h_{1} L\right)$,

or

$a(L)=\frac{a(0) \exp \left(h_{2} L\right) \exp \left(i h_{1} L\right)}{1-\epsilon \exp \left(h_{2} L\right) \exp \left(i h_{1} L\right)}$.

From Eq. (3), the amplitude feedback ratio $\epsilon$ must be larger than $1 / \exp \left(h_{2} L\right)$ to satisfy the oscillation condition. The threshold happens as the denominator of Eq. (3) vanishes, which implies a finite transmitted wave $a(L)$ may still take place spontaneously even without any incident source $a(0)$. It also requires $h_{1} L=2 \pi n$ to satisfy the phase matching condition with $n$ being an integer. Since $h_{1}$ and $h_{2}$ are dependent on $P$ and $Q$ only certain spatial and temporal frequency combination can satisfy both conditions simultaneously. In other words, only the noise of certain spatial and temporal frequencies satisfying the oscillation condition will get infinite amplification and emerges.

However, the oscillation condition of Eq. (3) is based on the "ideal feedback" assumption, i.e. the total optical path is a multiple of the wavelength. If there is a phase deviation caused by the difference between the length of the feedback optical path and the multiple of the wavelength, it will bring an additional phase to the field and modify Eqs. (2) and (3), respectively to

$a(L)=[a(0)+\epsilon a(L) \exp (i \delta)] \exp \left(h_{2} L\right) \exp \left(i h_{1} L\right)$,

and

$a(L)=\frac{a(0) \exp \left(h_{2} L\right) \exp \left(i h_{1} L\right)}{1-\epsilon \exp \left(h_{2} L\right) \exp \left[i\left(h_{1} L+\delta\right)\right]}$.

The new oscillation conditions, hence, are rewritten as $h_{1} L+\delta=2 n \pi$ and $1-\epsilon \exp \left(h_{2} L\right)=0$.

\section{Experimental setup}

We then try to observe the theoretical prediction experimentally. We conduct the experiment with a biased photorefractive SBN:60 crystal $(a \times b \times c=5 \mathrm{~mm} \times 5 \mathrm{~mm} \times 10 \mathrm{~mm})$ inside the cavity (Fig. 2). A collimated laser light with extraordinary polarization from a $532 \mathrm{~nm}$ CW double frequency NdYAG laser passes through the SBN crystal and covers the entire crystal. To ensure the meanfield statement, we performed another interference experiment to make sure its coherent length is several times the length of the cavity and it is a single mode. PBS1 reflects part of the light for the feedback. The amount of feedback can be controlled by adjusting WP1. We adopt the 4-f image method twice by inserting four lenses in the feedback path to project the image at the output face of the crystal onto the input face. WP2 and PBS2 rotate the polarization of the feedback light beam to be extraordinary while BS in front of the crystal combines the incident and feedback beams. BS is well adjusted to make sure the image at the output face of the crystal is projected at the input face with exactly the same transverse coordinates. The angle between the overlapping light beams is also adjusted to be smaller than $1.2 \times 10^{-4}$ degree to make sure the fields are added as parallel plane waves. To create the selfdefocusing condition, a bias voltage is applied alone the $c$-axis of the crystal. Finally, all experimental images are captured by a lens and a CCD camera. In this setup, the total optical path of the feedback is less than $1 \mathrm{~m}$, much less than the coherence length of the $\mathrm{cw}$ laser light, ensuring the mean field assumption.

\section{Experimental results}

We start the experiment with a biasing voltage at $300 \mathrm{~V}$, which yields a maximal index change of $\Delta n=5.06 \times 10^{-5}$ given that the effective electro-optic coefficient is $260 \mathrm{pm} / \mathrm{V}$. The illumination intensity is $39 \mathrm{mw} / \mathrm{mm}^{2}$. We increase the feedback ratio $(\epsilon)$ gradually from zero. There is no spontaneous pattern formation for the feedback ratio up to $16 \%$ (Fig. 3a). But when the feedback is near the threshold at around $22 \%$, the pattern begin to emerge spontaneously (Fig. 3b). When it is well above the threshold, the patterns are clearly displayed (Fig. 3c). According to the theoretical analysis, the threshold value for $\epsilon$ is proportional to $1 / \exp \left(h_{2} L\right)$, where $h_{2}$ relates positively to the nonlinearity strength $\kappa$, meaning a system with smaller $\kappa$ needs larger feedback to satisfy the oscillation con- 


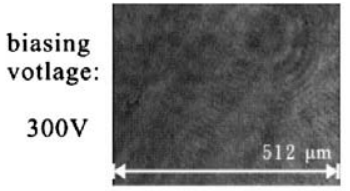

(a) $16 \%$

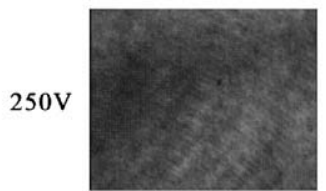

(d) $27 \%$

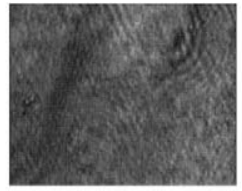

(b) $22 \%$

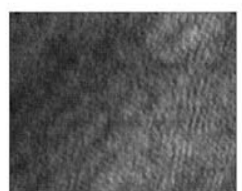

(e) $34 \%$

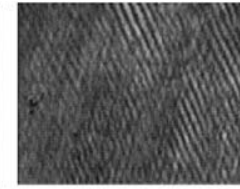

(c) $26 \%$

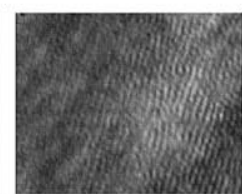

(f) $36 \%$
Fig. 3. Light intensity patterns at the output face of the self-defocusing photorefractive SBN crystal under different biasing voltage within a cavity of different feedback ratios. Note the faint stripes shown in (a) and (d) are due to the striation in the crystal.

dition. Fig. 3d-f shows another series of results under the biasing voltage $250 \mathrm{~V}$ and the intensity $26 \mathrm{mw} / \mathrm{mm}^{2}$. Since the nonlinearity strength of this arrangement for photorefractive material depends on the applied voltage only [23-26], we can see that the threshold feedback required in the second case is about 34\% indeed much larger than $22 \%$ in the first case. We also notice that the patterns, when appear, are not static but continue to vary with time (Fig. 4). This is predicted by the theory shown in Fig. 1, in which noise component with $\Omega=0$ obtains zero gain. Moreover, the pattern with higher feedback ratio has the faster moving rate. This is because higher feedback yields higher total intensity and the photorefractive response time is inversely proportional to the total intensity. Fig. 4 shows the dynamic pattern of feedback ratio of $26 \%$. By measuring the number of stripes passing through a fixed point in a specific time interval, we can estimate the temporal frequency of the moving patterns. The corresponding temporal frequency of Fig. $6(\epsilon=26 \%)$ is $0.016 \mathrm{~s}^{-1}$ per second compared to $0.013 \mathrm{~s}^{-1}$ when the feedback is $22 \%$.

Even under the same $\epsilon$, patterns of different spatial periods appear at different time (Fig. 5). In our experiment, since the optical path drifts very slowly within a small fraction of wavelength, this results in the phase deviation during the feedback process. The form of phase matching condition is $h_{1} L+\delta=2 n \pi$ as the path deviation is taken into account. For keeping the phase matching condition, $h_{1}$ must vary with $\delta$. Because $h_{1}$ is the function of spatial

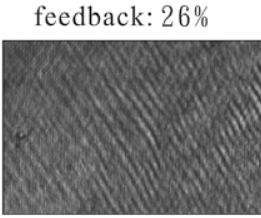

$\mathrm{t}=0(\mathrm{~s})$

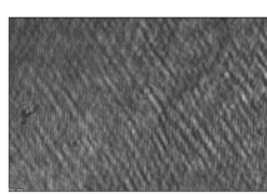

6

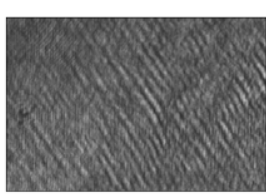

12
Fig. 4. Time varying MI patterns. (1.95 MB).

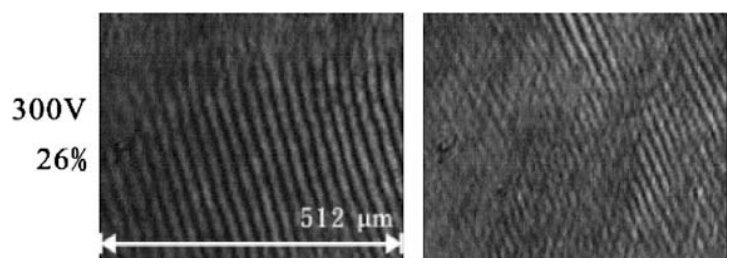

Fig. 5. Formation of MI patterns of different spatial periods under the same feedback ratio and strength of the nonlinearity at the same area at different time.

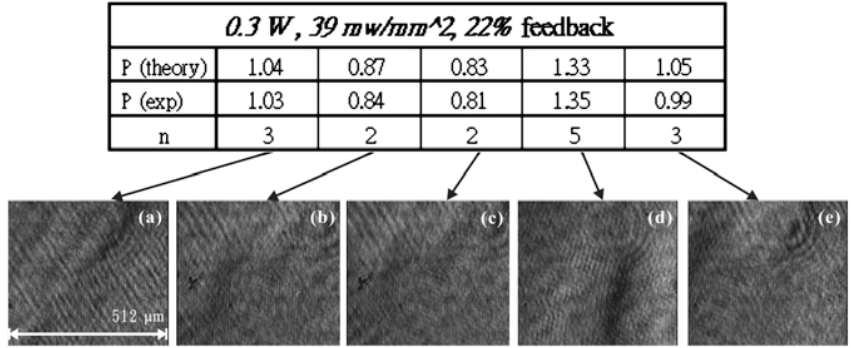

Fig. 6. Table of normalized spatial frequency $P . P(\exp )$ is measured from the experimental patterns. $P$ (theory) is derived from theory.

and temporal frequency, different phase deviation $\delta$ then leads to different spatial period. To confirm this, we do some quantitative calculations to compare it with the theory. Although the phase shift $\delta$ is not controlled in the experiment, we still can estimate it. The total field leaving the cavity is the summation of all fields inside, including the incident light $E_{0}$ and the additional part from feedback. If the feedback factor $\epsilon$ is small enough, the total intensity can be expressed as $I=\sqrt{\left(E_{0}+\epsilon E_{0} \cos \delta\right)^{2}+\left(\epsilon E_{0} \sin \delta\right)^{2}} \approx$ $(1+2 \epsilon \cos \delta)\left|E_{0}\right|^{2}=(1+2 \epsilon \cos \delta) I_{0}$ to the first order of $\epsilon$. By measuring the total intensity with the feedback $(I)$ and without it $\left(I_{0}\right)$, we can obtain $\cos \delta$ and $\delta$ corresponding to every experimentally measured feedback ratio $(\epsilon)$, i.e. $\cos \delta=\frac{\left(I / I_{0}\right)-1}{2 \epsilon}$, and evaluate $h_{1}$ through the phase matching condition $h_{1} L+\delta=2 n \pi$ by indicating certain integer of $n$. We know $h_{1}$ is related to $h_{2}$ of the form $h_{1}=-\left[\frac{\kappa k}{n_{0}} A_{0}^{2}\right]^{2} \frac{Q}{1+Q} \frac{P^{2}}{h_{2}}$. At exactly the threshold, there is a corresponding $h_{2}$ to satisfy the oscillation condition $\epsilon=1 / \exp \left(h_{2} L\right)$. Therefore, we can estimate $h_{2}=-\ln \epsilon / L$ and get a new $P$-Q-relation of $\frac{Q P^{2}}{1+Q}=h_{1} h_{2} /-\left[\frac{\kappa k}{n_{0}} A_{0}^{2}\right]^{2}$. Together with the equation $h_{2}=\frac{k k}{\sqrt{2} n_{0}} A_{0}^{2} \sqrt{-P^{4}-\frac{2 P^{2}}{1+Q^{2}}+P^{2} \sqrt{P^{4}+\frac{4+4 P^{2}}{1+Q^{2}}}}$, we can find out the theoretically allowed normalized spatial frequency $P$ (theory). $P(\exp )$ can directly be obtained based on the definition of the normalized spatial frequency $P=k_{\perp} /\left[\left(2 \kappa / n_{0}\right)\right]^{1 / 2} \cdot k A_{0}$ where $k_{\perp}$ is the distance from the origin to the peak of the Fourier transform of the intensity picture corresponding to the spatial period. In both $P$ (theory) and $P(\exp ), \kappa \times A_{0}^{2}=\Delta n$ is used. We operate the experiment just at the threshold feedback under biasing voltage at $300 \mathrm{~V}$. We observe the patterns and measure the total intensity $I$ and find them to change with respect to time. We calculate their corresponding $P$ (theory) and $P(\exp )$, and compare them with the captured experimental images from CCD. We find that theoretical and experimental results agree very well (Fig. 6). The spatial period varies at different moment with different corresponding $\delta$. It never really reach as a steady state. Most time it oscillates between $n=2$ or $n=3$, but sometimes at the higher order $n=5$. Also found in the results, larger $n$ corresponds to smaller spatial periods. This is similar to a laser cavity, in which larger round-trip phase yields shorter wavelength.

\section{Conclusion}

In conclusion, we investigate the modulation instability in the noninstantaneous defocusing medium with a coherent feedback theoretically and experimentally. We find out the instability will happen spontaneously if the feedback exceeds a certain threshold. For the larger biasing voltage, which generates the higher nonlinearity, the threshold is lower. The phase matching condition of the coherent feedback decides the spatial periods of the MI pat- 
terns. This research is supported by National Science Council, Taiwan.

\section{References}

[1] E. Infeld, G. Rowlands, Nonlinear Waves Solitons and Chaos, Cambridge University Press, 2000.

[2] G.P. Agrawal, Nonlinear Fiber Optics, Academic Press, 1995.

[3] M. Remoissenet, Wave Called Solitons, Springer-Verlag, 1999.

[4] M.J. Lighthill, Proc. Roy. Soc. Lond. A 299 (1967) 28.

[5] S.J. Bentley, R.W. Boyd, W.E. Butler, A.C. Melissinos, Opt. Lett. 26 (2001) 1084.

[6] M. Shih, F. Sheu, PRL 86 (2001) 2281.

[7] R.Y. Chiao, P.L. Kelley, E. Garmire, PRL 17 (1966) 1158.

[8] M. Shih, C. Jeng, F. Sheu, C. Lin, PRL 88 (2002) 133902.

[9] J. Miguel Hickmann, A.S.L. Gomes, Cid B. de Araujo, PRL 68 (1992) 3547

[10] D. Michaelis et al., PRA 56 (1997) R3366.

[11] W.H. Chu, C.C. Jeng, C.H. Chen, Y.H. Liu, M.F. Shih, Opt. Lett. 30 (2005) 1846

[12] T. Carmon, M. Soljacic, M. Segev, PRL 89 (2002) 183902.
[13] S.J. Bentley, R.W. Boyd, W.E. Butler, A.C. Melissinos, Opt. Lett. 26 (2001) 1084

[14] Damia Gomila, Roberta Zambrini, Gian-Luca Oppo, Opt. Lett. 92 (2004) 253904.

[15] L.A. Lugiato, R. Lefever, PRL 58 (1978) 2209.

[16] M. Schwab, C. Den, M. Saffman, JOSAB 299 (2001) 628.

[17] O. Sandfuchs, F. Kaiser, M.R. Belic, PRA 64 (2001) 063809.

[18] J.B. Geddes, R.A. Indik, J.C. Meloney, PRA 50 (1994) 3471.

[19] G. D’Alessandro, W.J. Firth, PRL 66 (1991) 2597.

[20] T. Nagaya, T. Yamamoto, et al., JOSAB 25 (2008) 74.

[21] R. Neubecker, G.L. Oppo, B. Thuering, T. Tschudi, PRA 52 (1995) 791.

[22] F.T. Arecchi, S. Boccaletti, S. Ducci, E. Pampaloni, P.L. Ramazza, S. Residori, J. Nonlinear Opt. Phys. Mater. 9 (2000)

[23] R.W. Boyd, Nonlinear Optics, Photorefractive Equations of Kukhtarev et al., Sec 10, Academic Press, 1992.

[24] M. Mitchell, Z. Chen, M. Shih, M. Segev, PRL 77 (1996) 490.

[25] P. Zhang, J. Zhao, C. Lou, X. Tan, Y. Gao, Q. Liu, D. Yang, J. Xu, Z. Chen, Opt. Exp. 15 (2007) 536

[26] S. Jia, W. Wan, J.W. Fleischer, Opt. Lett. 32 (2007) 1668. 